\title{
Evaluation of Apparent Diffusion Coefficients in the Cerebellar Tonsils and Bulbus in Chiari Type I Malformations: Comparison Before and After Surgery
}

\author{
Bekir AKGUN¹, Sait OZTURK', Ismail TASKENT², Mehmet Besir SURME¹, Fatih Serhat EROL ${ }^{1}$, Hanefi YILDIRIM² \\ ${ }^{1}$ Firat University, School of Medicine, Department of Neurosurgery, Elazig, Turkey \\ ${ }^{2}$ Firat University, School of Medicine, Department of Radiology, Elazig, Turkey
}

\section{ABSTRACT}

AIM: To evaluate the preoperative and postoperative $6^{\text {th }}$ month mean apparent diffusion coefficient (ADC) values of the cerebellar tonsils and bulbus in patients with Chiari Malformation Type I (CMI), and to compare the results with healthy controls.

MATERIAL and METHODS: We included 15 patients with CMI who underwent suboccipital decompression, upper cervical laminectomy, and duraplasty surgery, and compared them with 10 healthy individuals. Three regions of interest were placed, one each in the cerebellar tonsils and one in the bulbus. The mean ADC values were measured separately in each region.

RESULTS: Among the patients, mean ADC values were significantly decreased after surgery compared with before surgery. The mean ADC values before surgery were significantly higher for patients than for controls; however, although the mean ADC values were slightly higher after surgery for patients than for controls, the differences were not significant. Thus, after surgical intervention, $\mathrm{ADC}$ values in patients with $\mathrm{CMI}$ became close to those of normal individuals.

CONCLUSION: The increased ADC values in patients with CMI before surgery implied that not only morphologic changes but also increased diffusivity may play a key role in the pathophysiology and clinical presentation of the disease. Decompression surgery can produce favorable diffusional alterations.

KEYWORDS: Apparent diffusion coefficient, Chiari malformation type 1, Diffusion-weighted imaging, Suboccipital decompression

\section{INTRODUCTION}

$\mathrm{T}$ Type 1 Chiari malformation (CMI) is the most common Chiari malformation, and is characterized by caudal displacement of the cerebellar tonsils into the cervical canal through the foramen magnum. The clinical signs and symptoms of $\mathrm{CMI}$ are believed to be manifestations of these anatomical changes. Consistent with this, patients with $\mathrm{CMI}$ exhibit various clinical signs and symptoms, due to cerebellar signs, brainstem compression, cranial nerve dysfunction, and spinal cord injury. Prominent examples include head, neck, back, and limb pain, as well as limb movement disorders, spasticity, sensory disturbances, vertigo, ataxia, nystagmus, and dysphagia $(5,9,16)$.
Computerized tomography and conventional magnetic resonance imaging (MRI) have been used to show many structural alterations that can either develop because of, or accompany, CMI. These include cerebellar tonsillar herniation, small posterior fossa volume, basilar invagination, occipitalization of the atlas, hydrocephalus, and syringomyelia $(4,9,15)$. In addition, many specific morphometric findings have been reported, such as a shallow posterior cranial fossa, short anteroposterior length of the foramen magnum, and a large angle of the tentorium (4). Although correlations have been confirmed between these structural alterations and clinical presentations in various studies, the degree of tonsillar herniation has not always been compatible with the 
clinical findings. Otherwise asymptomatic individuals who had incidentally detected cerebellar tonsillar herniation on $\mathrm{MRI}$ were encountered. There are also patients with persistent symptoms despite decompression surgery $(7,14)$. Therefore, we considered that not only anatomical changes but also some other pathophysiological factors may be important in the development of clinical signs and symptoms.

Conventional MRI can fail to depict physiological changes of some diseases and novel modalities have therefore been developed. Diffusion-weighted imaging (DWI) is one such method that is based on the random movement of water molecules in tissue, relying on the rate of net molecule diffusion to give an apparent diffusion coefficient (ADC) value (8). Such quantitative neuroimaging indices have helped to advance our understanding of the underlying pathophysiologic mechanisms of many neurological diseases (1).

In this study, we aimed to improve our understanding of the pathophysiological and microstructural characteristics of CMI.

\section{MATERIAL and METHODS}

We retrospectively evaluated patients who underwent surgery for CMI between January 2011 and September 2016. Mean ADC values were measured from the cerebellar tonsils and bulbus before and 6 months after surgery. We then compared their results with measurements taken in healthy individuals who had normal cranial MRI scans.

\section{Patients}

The study group consisted of 15 patients with CMI (8 females, 7 males; mean age: 44 years, range: $26-54$ years). Of these, 8 patients had tonsillar herniation down to the $\mathrm{C} 1$ vertebra, and 7 patients had tonsillar herniation down to the $\mathrm{C} 2$ vertebra. All patients underwent suboccipital decompression (SOD), upper cervical laminectomy (UCL) based on cerebellar tonsillar herniation degree (C1 or $\mathrm{C} 1$ + partial $\mathrm{C} 2)$, and duraplasty surgery. Representative preoperative and postoperative $6^{\text {th }}$ month conventional MRI sections of a patient are presented in Figure 1A, $B$.
Patients who had hydrocephalus or who needed occipitocervical fusion due to an accompanying upper cervical vertebral abnormality were excluded. Patients with syringomyelia were included if the syrinx cavity was stable, downsized, or disappeared after surgery (SOD, UCL, and duraplasty); by contrast, we excluded patients who needed additional surgery for syringomyelia. In total, 12 patients had syringomyelia on admission, of whom 9 and 3 were affected in the cervical spinal cord and cervicothoracic junction, respectively. Of these, 2 remained stable, 7 downsized, and 3 disappeared at the MRIs of the postoperative $6^{\text {th }}$ months. The demographic features and preoperative conventional MRI findings of the patients are summarized in Table I.

\section{Radiological Evaluation}

MRls were performed using a 1.5 Tesla (General Electric, GE Healthcare, Chicago, IL, USA) MRI device with the following features: coils, 8-channel neurovascular diffusion, perfusion, spectroscopy, and tractography. The DWIs and the ADC values were evaluated simultaneously by two radiologists and two neurosurgeons. DWIs were taken before and at 6 months after surgery for all 15 cases. For comparison, we used the data for 10 healthy individuals (controls) with normal cranial MRIs (1000 gradient $b$ values). The obtained DWIs were processed on the MRI machines study station, using Advantage Windows, Version 4.6 (GE Medical Systems), and color ADC maps of the brain parenchyma were produced. Three regions of interest (ROIs) were placed, one each in the bilateral central parts of the cerebellar tonsils and one in the anterior part of the bulbus (Figure 2A-D). Mean ADC values were measured separately and automatically $\left(\mathrm{s} / \mathrm{mm}^{2}\right)$. We used the same ROI sizes in the patients and controls $\left(30 \mathrm{~mm}^{2}\right.$ for the cerebellar tonsils, and $60 \mathrm{~mm}^{2}$ for the bulbus). When considering the morphology of the tonsils, measurements were performed symmetrically from the central part of the tonsil parenchyma to reduce artifacts. When performing measurements of the bulbus, the anterior parts were preferred to avoid cerebrospinal fluid (CSF) artifacts.
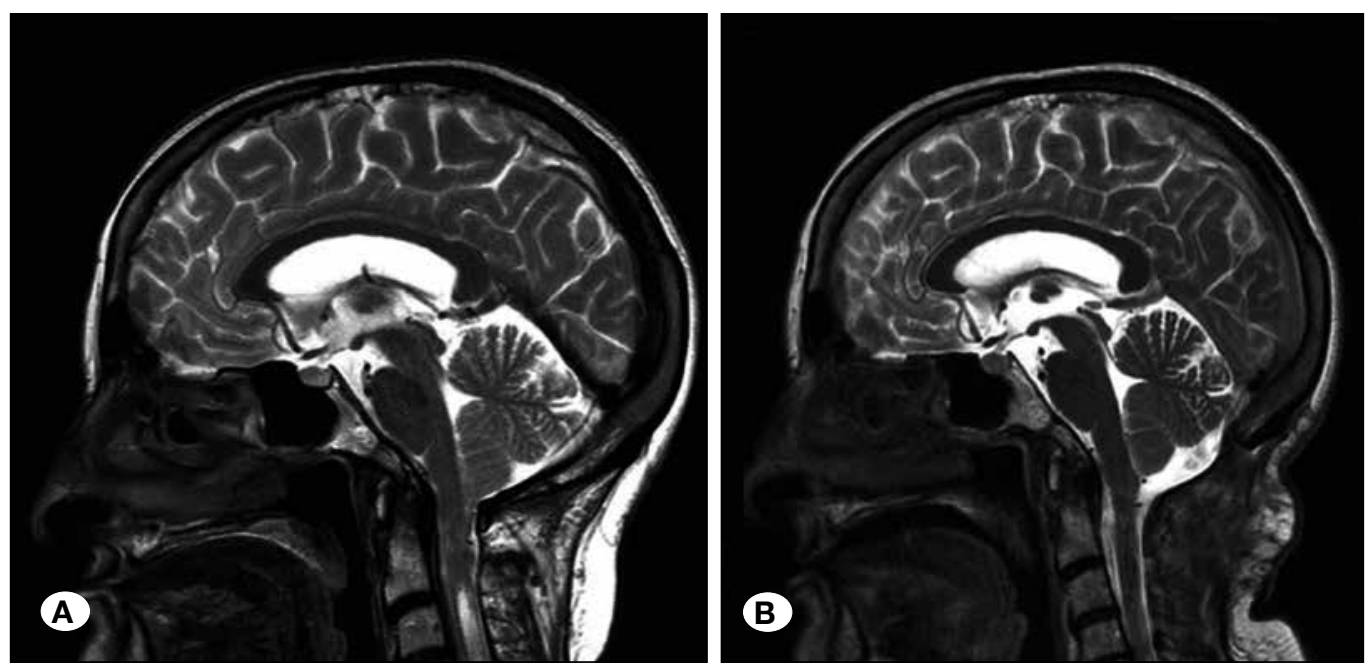

Figure 1: Representative T2 weighted sagittal MRls of a patient.

A) Before surgery, crowding of the foramen magnum by cerebellar tonsillar herniation, extending to the upper $\mathrm{C} 2$ vertebral level and the upper part of a syringomyelia starting at the C3 level; B) After surgery, foramen magnum expansion and disappearance of the syringomyelia are shown. 


\section{Statistical Analysis}

Data were analyzed using IBM SPSS for Windows, Version 22.0 (IBM Corp., Armonk, NY, USA). Means and standard deviations were used when reporting descriptive statistics. Differences in the repetitive measurements before and after surgery were analyzed with the Wilcoxon test. The MannWhitney $U$ test was used to compare quantitative continuous data between two independent groups (patients and controls). All findings were evaluated based on $95 \%$ confidence intervals, and tests were considered statistically significant at $p<0.05$.

\section{ם RESULTS}

Mean ADC values of the patients decreased significantly from before to 6 months after surgery in all three regions (i.e., right tonsil, left tonsil, and bulbus). Moreover, the postsurgical values became close to those of the control group. In the patient group, differences between the mean values from before to 6 months after surgery were also statistically significant (Wilcoxon test) $(\mathrm{p}=0.005)$ (Table II). Thus, high ADC values before surgery were reduced after suboccipital decompression surgery (Figure 3).

Differences in the mean preoperative measurements between patients and controls were statistically significant with the Mann-Whitney $U$ test. In all three regions, mean ADC values for patients before surgery were significantly higher than for controls $(p=0.000$ )(Table III). However, although postoperative

Table I: Demographic Features and Conventional MRI Findings of the Patients

\begin{tabular}{lccccccc}
\hline Age (year) & \multicolumn{2}{c}{ Gender } & \multicolumn{2}{c}{$\begin{array}{c}\text { Degree of Tonsillar } \\
\text { Herniation }\end{array}$} & \multicolumn{2}{c}{ Coexistence of Syringomyelia } \\
\hline $\begin{array}{l}26-54 \\
\text { (mean: } 44)\end{array}$ & Female & Male & $\begin{array}{c}\text { Down to C1 } \\
\text { vertebra }\end{array}$ & $\begin{array}{c}\text { Down to C2 } \\
\text { vertebra }\end{array}$ & Cervical & Cervicothoracic & Absent \\
\cline { 2 - 8 } & 8 patients & 7 patients & 8 patients & 7 patients & 9 patients & 3 patients & 3 patients \\
\hline
\end{tabular}
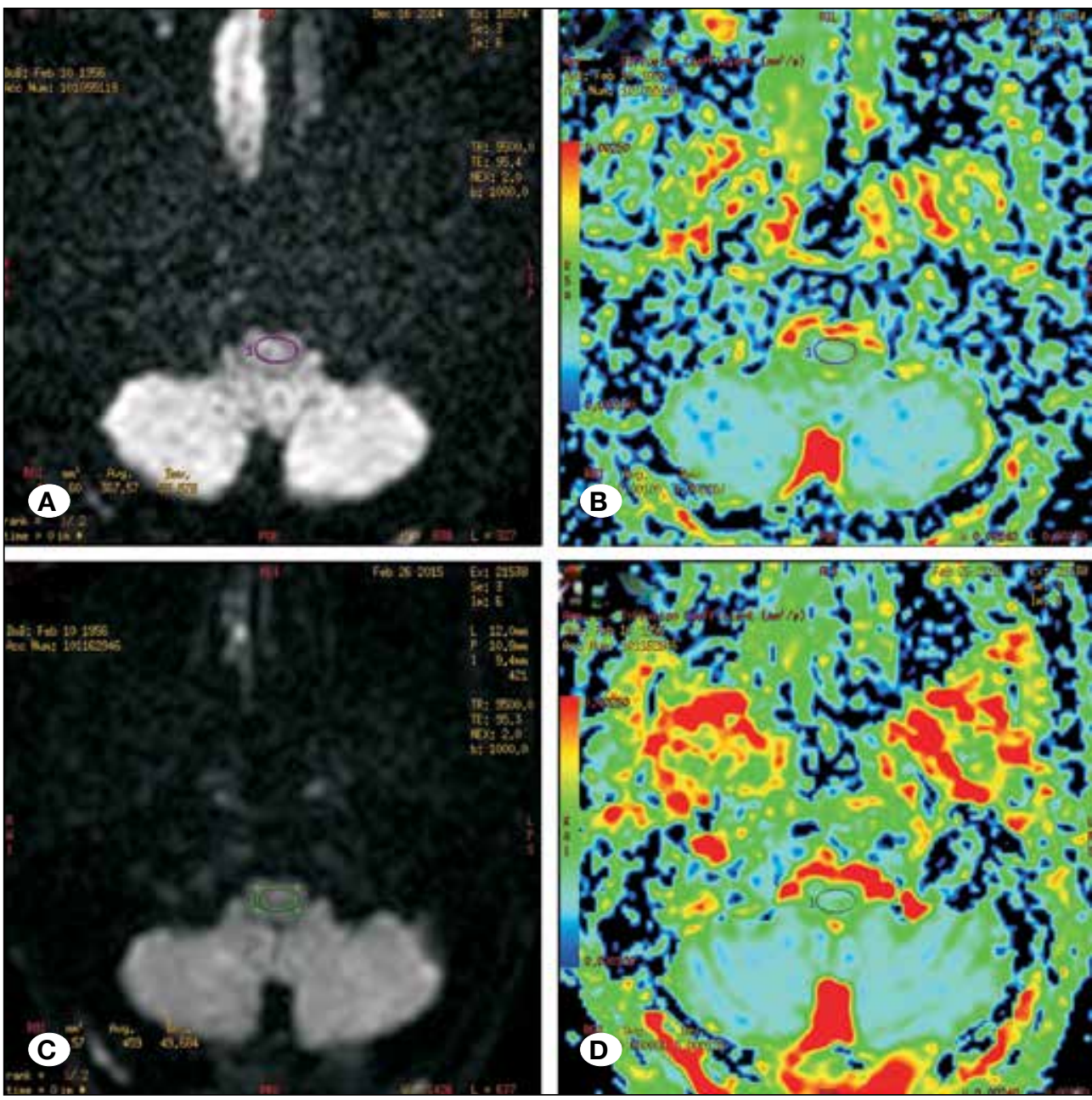

Figure 2: A patient's

representative axial images showing ROls in the bulbus.

A) DWI before surgery,

B) ADC mapping before surgery,

C) DWI after surgery, and

D) ADC mapping after surgery. 
$6^{\text {th }}$ month mean ADC values of the patients were slightly higher than the controls, the differences were not significant $(p>0.05)$ (Table IV). Thus, the ADC values of the cerebellar tonsils and bulbus increased in patients with CMI. However, the values reached approximately those of normal individuals after SOD, UCL, and duraplasty (Figure 4).

\section{DISCUSSION}

Structural studies and conventional images may not always provide sufficient information in patients with CMI. Physiological or microstructural examination may be necessary for two reasons. First, the presence and severity of clinical symptoms do not always correlate with the degree of tonsillar herniation, the dimensions of the posterior fossa, the extent of syringomyelia, or other morphological features $(2,13)$. Second, despite technically successful decompression surgery, some patients have persistent symptoms, with conventional MRIs often showing realized decompressions of the posterior fossa for these patients $(2,7)$.

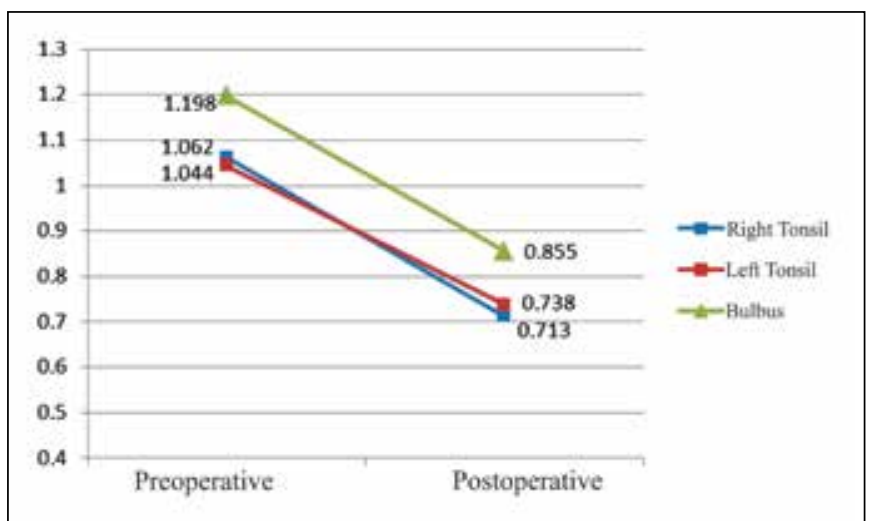

Figure 3: Comparison of the mean ADC values of patients from before to the $6^{\text {th }}$ month after surgery.
Current advanced MRI techniques, such as diffusion tensor imaging (DTI) and CSF flow studies, allow for a better understanding of the complex underlying pathophysiology of CMI $(7,9,13,16)$. DTI, for example, provides information about diffusion restriction and neuronal microstructural integrity. Studies have shown that diffusion restriction is especially notable before surgery in the anterior brainstem compartment of patients with CMI. Moreover, it has been shown that this may be normalized by decompression surgery (7). Also not only CMI, Woitek et al. determined elevated fractional anisotropy in the midbrain in fetuses with Chiari II malformation by fetal diffusion tensor quantification of brainstem pathology. These findings are thought to be caused by changes in the internal architecture of the brainstem, supposedly resulting from external compression (17).

Studies utilizing phase-contrast MRI have also shown that obstruction of the foramen magnum causes abnormal CSF flow, especially at the C2-3 level. Such CSF flow studies are now being used to evaluate and predict outcomes in patients with CMI before and after surgery; however, no standardized protocols are currently available $(9,15,16)$. Because of the complex anatomy of the craniospinal junction and the differences in CSF flow dynamics between individuals, CSF flow studies may not always provide reliable information. Indeed, CSF flow can be affected by various local and systemic factors (13). New assessment modalities that are less affected by such factors are therefore needed if we want to evaluate the functional results of decompression surgery accurately. In this context, DWI with ADC examinations may be appropriate.

Alterations in permeability, osmolarity, or active transportation may cause physical shifts in the number of water protons in tissue compartments, which can be observed by DWI (12). In both clinical and experimental studies of ischemic incidents, it has been noted that DWI sequences in affected regions show decreased diffusivity and decreased ADC values in the acute stages because of decreases in extracellular water. However,

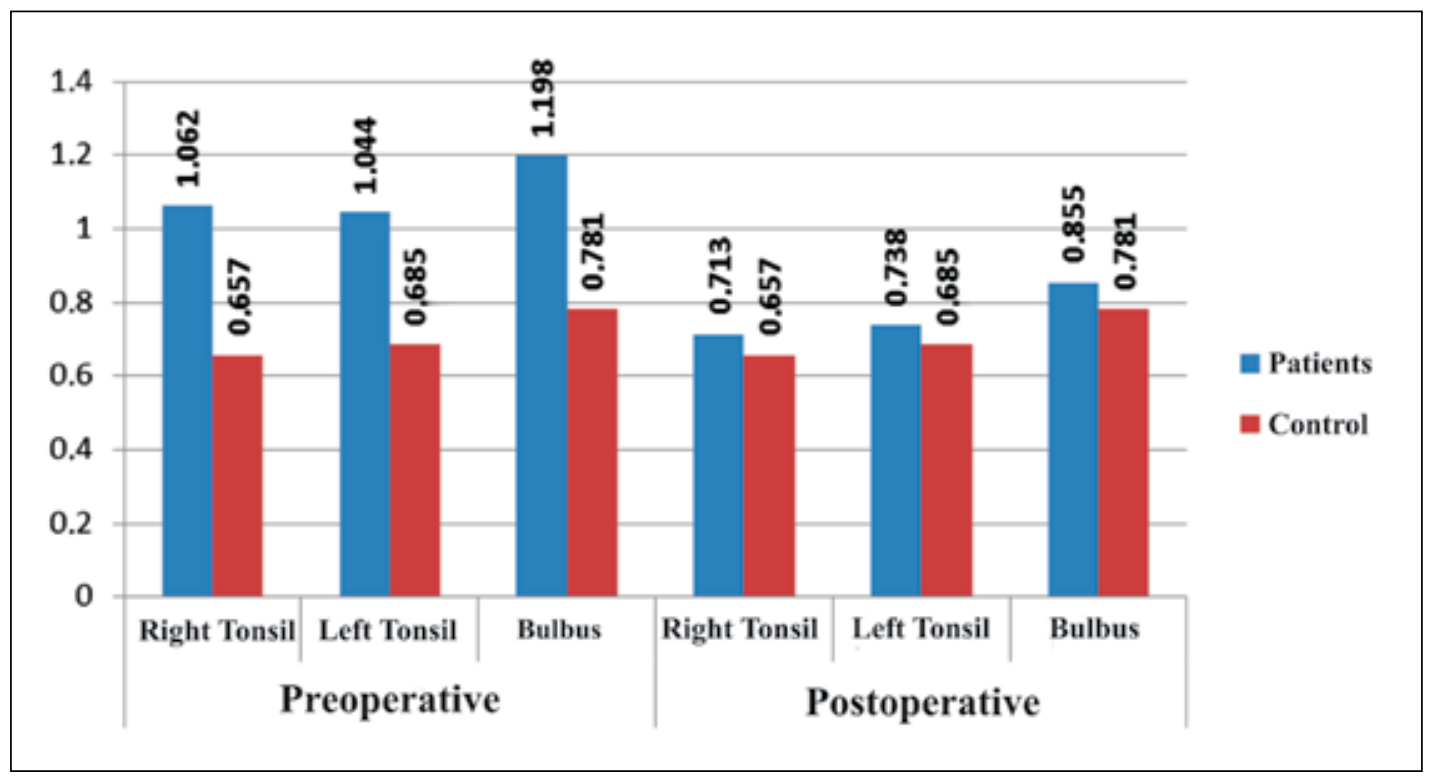

Figure 4: Comparison of the mean ADC values between patients and healthy controls. 
during chronic stages, the same regions show increased $A D C$ values because of the presence of increased diffusion with increased extracellular fluid content $(1,6)$. Similar results have been obtained when measuring ADC values in traumatic brain injury: in the early phase, mean ADC values of injured regions are mostly decreased, but these tend to increase as the injury becomes chronic. Some studies even showed a linear trend, with a gradual increase in the mean ADC value from early to chronic phases (11). A correlation between increasing injury severity and increasing ADC values has also been reported. Pathophysiological mechanisms that may explain diffuse increases in the ADC value include vasogenic edema, chronic ischemic phenomena, and changes in either the tissue cytoarchitecture or neurofilament alignment (3).

We were unable to identify any study that evaluated the posterior fossa ADC values of patients with CMI. However, Mignone Philpott et al. performed a diffusion-weighted MRI investigation in fetuses with Chiari II malformations, and they reported that the cerebellar ADC values were, on average, $35 \%$ higher than those of healthy fetuses. They attributed this increased diffusivity to either the altered CSF hydrodynamics in the posterior fossa or to the indirect effects of such dynamics on membrane and fiber composition (10).

In our study, the preoperative mean ADC values of patients with $\mathrm{CMl}$ were significantly higher than those of healthy control individuals. According to this and previous research, high mean ADC values before surgery in patients with $\mathrm{CMI}$ may be caused by pathophysiological mechanisms, including chronic ischemic phenomena, vasogenic edema, altered CSF hydrodynamics, and effects on fiber composition caused by chronic compression of the posterior fossa. After surgical elimination of the chronic compression, we found that mean ADC values decreased to levels that approximated those of healthy control individuals. This likely contributed to improvement of the pathophysiological incidents typical of posterior fossa decompression. However, although we obtained favorable quantitative and physiological neuroimaging results after decompression surgery, we did not evaluate the correlation between the ADC values and either the degree of tonsillar herniation (or other structural changes) or the severity of clinical

Table II: Preoperative and Postoperative Mean ADC Values of Patients and "p" Values

\begin{tabular}{|c|c|c|c|}
\hline \multicolumn{4}{|c|}{ ADC values $\left(\times 10^{-3} \mathrm{~mm}^{2} / \mathrm{sec}\right)$} \\
\hline ROls & Preoperative (mean \pm sd) & Postoperative (mean \pm sd) & p value \\
\hline Right Tonsil & $1.062 \pm 0.189$ & $0.713 \pm 0.084$ & 0.005 \\
\hline Left Tonsil & $1.044 \pm 0.171$ & $0.738 \pm 0.070$ & 0.005 \\
\hline Bulbus & $1.198 \pm 0.290$ & $0.855 \pm 0.117$ & 0.005 \\
\hline
\end{tabular}

ADC: Apparent Diffusion Coefficient, ROI: Region of interest, sd: Standard deviation.

Table III: Differences Between Preoperative Mean ADC Values of Patients and Mean ADC Values of Healthy Individuals

\section{$\operatorname{ADC}\left(\times 10^{-3} \mathrm{~mm}^{2} / \mathrm{sec}\right)$}

\begin{tabular}{lccc}
\hline ROIs & $\begin{array}{c}\text { Patients (Preoperative) } \\
\text { mean } \pm \text { sd }\end{array}$ & $\begin{array}{c}\text { Control (Healthy individuals) } \\
\text { mean } \pm \text { sd }\end{array}$ & p value \\
\hline Right Tonsil & $1.062 \pm 0.189$ & $0.657 \pm 0.030$ & 0.000 \\
\hline Left Tonsil & $1.044 \pm 0.171$ & $0.685 \pm 0.067$ & 0.000 \\
\hline Bulbus & $1.198 \pm 0.290$ & $0.781 \pm 0.073$ & 0.000 \\
\hline
\end{tabular}

ADC: Apparent Diffusion Coefficient, ROI: Region of interest, sd: Standard deviation.

Table IV: Differences Between Postoperative Mean ADC Values of Patients and Mean ADC Values of Healthy Individuals

$\operatorname{ADC}\left(\times 10^{-3} \mathrm{~mm}^{2} / \mathrm{sec}\right)$

\begin{tabular}{lccc}
\hline ROIs & $\begin{array}{c}\text { Patients (Postoperative) } \\
\text { mean } \pm \text { sd }\end{array}$ & $\begin{array}{c}\text { Control (Healthy individuals) } \\
\text { mean } \pm \text { sd }\end{array}$ & p value \\
\hline Right Tonsil & $0.713 \pm 0.084$ & $0.657 \pm 0.030$ & 0.112 \\
\hline Left Tonsil & $0.738 \pm 0.070$ & $0.685 \pm 0.067$ & 0.034 \\
\hline Bulbus & $0.855 \pm 0.117$ & $0.781 \pm 0.073$ & 0.059 \\
\hline ADC:App &
\end{tabular}

ADC: Apparent Diffusion Coefficient, ROI: Region of interest, sd: Standard deviation. 
symptoms, findings, or prognosis. This was because of the small sample size, and is an important limitation of our study.

\section{- CONCLUSION}

$A D C$ values of the cerebellar tonsils and bulbus that were exposed to chronic compression and constriction were significantly elevated in patients with CMI when compared with $A D C$ values in healthy controls. Furthermore, these values decreased six months after decompression and duraplasty surgery, becoming similar to those of healthy control individuals. Although promising for improving our understanding of the pathophysiology and the clinical signs and symptoms of CMl, our results need to be supported by additional physiological and functional imaging studies.

\section{REFERENCES}

1. Ahlhelm F, Schneider G, Backens M, Reith W, Hagen T: Time course of the apparent diffusion coefficient after cerebral infarction. Eur Radiol 12: 2322-2329, 2002

2. Alperin N, Loftus JR, Oliu CJ, Bagci A, Lee SH, Erti-Wagner $B$, Green B, Sekula R: MRI measures of posterior cranial fossa morphology and CSF physiology in Chiari Malformation Type I. Neurosurgery 75: 515-522, 2014

3. Goetz P, Blamire A, Rajagopalan B, Cadoux-Hudson T, Young $D$, Styles $P$ : Increase in apparent diffusion coefficient in normal appearing white matter following human traumatic brain injury correlates with injury severity. J Neurotrauma 21:645-654, 2004

4. Hwang HS, Moon JG, Kim CH, Oh SM, Song JH, Jeong JH: The comparative morphometric study of the posterior cranial fossa: What is effective approaches to the treatment of Chiari malformation type 1? J Korean Neurosurg Soc 54: 405-410, 2013

5. Imperato A, Seneca V, Cioffi V, Colella G, Gangemi M: Treatment of Chiari malformation: Who, when and how. Neurol Sci 32: 335-339, 2011

6. Karki K, Knight RA, Shen LH, Kapke A, Lu M, Li Y, Chopp M: Chronic brain tissue remodeling after stroke in rat: A 1-year multiparametric magnetic resonance imaging study. Brain Res 1360: 168-176, 2010

7. Krishna V, Sammartino F, Yee P, Mikulis D, Walker M, Elias $\mathrm{G}$, Hodaie $\mathrm{M}$ : Diffusion tensor imaging assessment of microstructural brainstem integrity in Chiari malformation Type I. J Neurosurg 125: 1112-1119, 2016
8. Le Bihan D, Breton E, Lallemand D, Grenier P, Cabanis $E$, Laval-Jeantet M: MR imaging of intravoxel incoherent motions: Application to diffusion and perfusion in neurologic disorders. Radiology 161: 401-407, 1986

9. McVige JW, Leonardo J: Neuroimaging and the clinical manifestations of Chiari Malformation Type I (CMI). Curr Pain Headache Rep 19: 18, 2015

10. Mignone Philpott C, Shannon P, Chitayat D, Ryan G, Raybaud CA, Blaser SI: Diffusion-weighted imaging of the cerebellum in the fetus with Chiari II malformation. AJNR Am J Neuroradiol 34: 1656-1660, 2013

11. Moen KG, Haberg AK, Skandsen T, Finnanger TG, Vik $A$ : A longitudinal magnetic resonance imaging study of the apparent diffusion coefficient values in corpus callosum during the first year after traumatic brain injury. J Neurotrauma 31: 56-63, 2014

12. Ozturk A, Oguz KK, Akalan N, Geyik PO, Cila A: Evaluation of parenchymal changes at the operation site with early postoperative brain diffusion-weighted magnetic resonance imaging. Diagn Interv Radiol 12: 115-120, 2006

13. Sivaramakrishnan A, Alperin N, Surapaneni S, Lichtor T: Evaluating the effect of decompression surgery on cerebrospinal fluid flow and intracranial compliance in patients with Chiari malformation with magnetic resonance imaging flow studies. Neurosurgery 55: 1344-1350, 2004

14. Stevens JM, Serva WA, Kendall BE, Valentine AR, Ponsford JR: Chiari malformation in adults: Relation of morphological aspects to clinical features and operative outcome. J Neurol Neurosurg Psychiatry 56: 1072-1077, 1993

15. Vurdem UE, Acer N, Ertekin T, Savranlar A, Inci MF: Analysis of the volumes of the posterior cranial fossa, cerebellum, and herniated tonsils using the stereological methods in patients with Chiari type I malformation. Scientific World Journal 2012: 616934, 2012

16. Wang CS, Wang X, Fu CH, Wei LQ, Zhou DQ, Lin JK: Analysis of cerebrospinal fluid flow dynamics and morphology in Chiari I malformation with cine phase-contrast magnetic resonance imaging. Acta Neurochir (Wien) 156: 707-713, 2014

17. Woitek R, Prayer D, Weber M, Amann G, Seidl R, Bettelheim D, Schöpf V, Brugger PC, Furtner J, Asenbaum U, Kasprian G: Fetal diffusion tensor quantification of brainstem pathology in Chiari II malformation. Eur Radiol 26: 1274-1283, 2016 